\title{
THE POTENTIAL ROLE OF HONEY AND ITS POLYPHENOLS IN PREVENTING HEART DISEASES: A REVIEW
}

\author{
Khalil M. I.*a, Sulaiman S. A.**b \\ Department of Pharmacology, School of Medical Sciences, Universiti Sains Malaysia, Health Campus, \\ 16150 Kubang Kerian, Kelantan, Malaysia. \\ E-mail: **Corresponding author: sbsamrah@kb.usm.my,*mibrahim12@yahoo.com
}

\begin{abstract}
Honey is rich in phenolic compounds, which act as natural antioxidants and are becoming increasingly popular because of their potential role in contributing to human health. A wide range of phenolic constituents is present in honey like quercetin, caffeic acid phenethyl ester (CAPE), acacetin, kaempferol, galangin which have promising effect in the treatment of cardiovascular diseases. Many epidemiological studies have shown that regular intake of phenolic compounds is associated with reduced risk of heart diseases. In coronary heart disease, the protective effects of phenolic compounds include mainly antithrombotic, anti-ischemic, anti-oxidant, and vasorelaxant. It is suggested that flavonoids decrease the risk of coronary heart disease by three major actions: improving coronary vasodilatation, decreasing the ability of platelets in the blood to clot, and preventing low-density lipoproteins (LDLs) from oxidizing. In this review paper, we discussed the preventive role of polyphenols of honey against cardiovascular diseases.
\end{abstract}

Key words: Honey; Antioxidant; Polyphenols; Cardiovascular diseases

\section{Introduction}

Antioxidant substances in produce and other foods may actually represent a modern-day "fountain of youth." Evidences suggest that vitamins $\mathrm{C}$ and $\mathrm{E}$, and beta-carotene, a precursor to vitamin $\mathrm{A}$, may reduce the risk of some forms of cancer, heart disease, strokes, and cataracts and may slow the aging process (National Honey Board, 2007). Honey is a remarkably complex natural liquid that is reported to contain at least 181 substances (White, 1975). The composition of honey is variable and primarily depends on the floral source; however, certain external factors may play a role also, such as seasonal and environmental factors and processing. Honey is a supersaturated solution of sugars, of which fructose $(38 \%)$ and glucose $(31 \%)$ are the main contributors. A wide range of minor constituents is also present in honey, many of which are known to have antioxidant properties (Ferreres et al., 1992; Andrade et al., 1997; Tan et al., 1989; Cherchi et al., 1994; White and Rudyj, 1978). The antioxidant activity of phenolic compounds might significantly contribute to the human health benefits of plant foods and beverages such as red wine and tea (Hertog et al., 1993; Bravo, 1998; Renaud and Lorgeril, 1992; Serafini et al., 1994).

Epidemiological data and studies with animal model point to a possible protective effect of flavonoids against cardiovascular diseases. Although flavonoids have been studied for about 50 years, the cellular mechanisms involved in their biological actions are still not completely known (Depeint et al., 2002). Many of the pharmacological properties of flavonoids can be linked to the abilities of these compounds to inhibit enzymes involved in cell activation. In vitro experiments demonstrated the capacity of flavonoids to modify the activity of enzymatic systems in mammals (kinases, phospholipases, ATPase, lipooxygenases, cyclooxygenases, phosphodiesterases etc.). A correlation has also been observed in some cases between the flavonoid structure and its enzymatic activity (Benavente-Garci'a et al., 1997; Marchend, 2002; Yanez et al., 2004; Bravo, 1998; Rodriguez et al., 2002; Martinez et al., 2003). Much of these effects can be attributed to the abilities of flavonoids to interact with the nucleotide-binding sites of regulatory enzymes. Research has also shown that flavonoids are potent radical scavengers and, thus, able to reduce many aging and degenerative events including reactive oxygen species (Benavente-Garc1'a et al., 1997). There is more information regarding the health benefits of honey but less information is available about the antioxidant properties of honey and its role against cardiovascular diseases. In this review, the discussion focuses on the antioxidant properties of honey and their ability to protect cardiovascular diseases.

\section{Antioxidant properties of honey}

"Gram for gram, antioxidants in buckwheat honey equals those of fruits and vegetables," said Dr. May Berenbaum, head of the University of Illinois' entomology department. "It packs the antioxidant power of Vitamin C in a tomato." Researchers at the University of Illinois-Champaign/Urbana have identified the antioxidant values of 14 unifloral honeys. The antioxidative components of honey were compared to an ascorbic acid standard. The water-soluble antioxidant content of the honey samples varied more than 20 -fold, from a high value of $4.32 \times 10^{-3}$ eq for Illinois buckwheat honey to a low value of $21.3 \times 10^{-5}$ eq for 
California button sage honey. Research showed a correlation between color and antioxidant capacity, with the darker honeys providing the highest levels of antioxidants. With antioxidant levels reaching $4.32 \times 10^{-3}$ meq., honey rivals those levels found in tomatoes $\left(2.83 \times 10^{-3} \mathrm{meq}\right)$ and sweet corn $\left(1.36 \times 10^{-3} \mathrm{meq}\right)$. Although honey by itself may not serve as a major source of dietary antioxidants, it demonstrates the potential for honey to play a role in providing antioxidants in a highly palatable form. Due to honey's pleasing taste, it may be more readily consumed by individuals reluctant to ingest plant-derived antioxidants. Certainly, compared to sucrose, which has no antioxidant value, honey can be a flavorful, supplementary source of antioxidants (National Honey Board: Food Technology Program, cited 2009).

Honey has been found to contain several antioxidant enzymes including glucose oxidase, catalase and other antioxidant components like ascorbic acid, flavonoids, phenolic acids, carotenoid derivatives, organic acids, Maillard reaction products, amino acids and proteins (Beretta et al., 2005; D'Arcy, 2005; Frankel et al., 1998; Aljadi and Kamaruddin, 2004; Inoue et al., 2005; Fahey and Stephenson, 2002; Blasa et al., 2006; Nagai et al., 2006 and Perez et al., 2007). Various polyphenols are reported in honey. Some of the polyphenols of honey like caffeic acid, caffeic acid phenyl ester, chrysin, galangin, quercetin, acacetin, kaempferol, pinocembrin, Pinobanksin and apigenin have evolved as promising pharmacological agents (Jaganathan and Mandal, 2009). Gheldof and Engeseth (2002) measured the antioxidative activity of honey polyphenols in vitro by comparing the oxygen radical absorbance capacity (ORAC) with the total phenolics concentration and showed that out of 14 honey samples Buckwheat Illinois honey have the highest value of ORAC and total phenolic content $(16.95 \mu \mathrm{mol}$ trolox equivalent/g and 796 gallic acid equivalent $/ \mathrm{kg}$ respectively). A significant correlation between the antioxidant activity, the phenolic content of honey and the inhibition of the in vitro lipoprotein oxidation of human serum was found by Gheldof et al. (2003). Furthermore, in a lipid peroxidation model system buckwheat honey showed a similar antioxidant activity as $1 \mathrm{mM} \alpha$-tocopherol. The influence of honey ingestion on the antioxidative capacity of plasma was tested in two studies (Schramm et al., 2003; Al-Waili, 2003). In the first one, the subjects were given maize syrup or buckwheat honeys with a different antioxidant capacity in a dose of $1.5 \mathrm{~g} / \mathrm{kg}$ body weight. In comparison to the sugar control, honey caused an increase of both the antioxidant and the reducing serum capacity. In the second study, humans received a diet supplemented with a daily honey of $1.2 \mathrm{~g} / \mathrm{kg}$ body weight. Honey increased the body antioxidant agents: blood vitamin $\mathrm{C}$ concentration by $47 \%, \beta$-carotene by $3 \%$, uric acid by $12 \%$, and glutathione reductase by $7 \%$. The antioxidant activity depends on the botanical origin of honey and varies to a great extent in honeys from different botanical sources (Baltrusaityte et al., 2007; Kücük et al., 2007 and Vela et al., 2007).

\section{Polyphenols and heart diseases}

Reactive oxygen species (ROS) are highly reactive molecules that are constantly produced by enzymatic reactions in cells. In normal physiological conditions, ROS are produced at low levels, which are necessary for maintaining normal cell functions, and the endogenous anti-oxidant defense systems of the body have the capacity to avert any harmful effects. However, several established risk factors for cardiovascular disease have been linked to excessive generation of ROS, known as a state of oxidative stress. For instance, in animal models of hiperlipidemia (Miller et al., 1998; Mugge et al., 1994), hypertension (Morawietz et al., 2001; Zalba et al., 2000; Suzuki et al., 1995), and diabetes (Hink et al., 2001; Sano et al., 1998), elevated levels of vascular superoxide anion production. Moreover, clinical studies have demonstrated that hypercholesterolemia and diabetes in humans are also associated with increased vascular superoxide anion generation (Guzik et al., 2000). All these data strongly suggest that increased oxidative stress is involved in the pathophysiology of cardiovascular disease.

Many epidemiological studies have shown that regular flavonoid intake is associated with a reduced risk of cardiovascular diseases (Middleton et al., 2000). In the coronary heart disease, the protective effects of flavonoids include mainly antithrombotic, antiischemic, anti-oxidant, and vasorelaxant (Jendekova et al., 2006). It is suggested that flavonoids decrease the risk of coronary heart disease by three major actions: (A) improving coronary vasodilatation, (B) decreasing the ability of platelets in the blood to clot and (C) preventing LDLs from oxidizing (Garc'ia and Castillo, 2008). Oxidation of low density lipoproteins is believed to play an important role in the development of atherosclerosis (Witztum and Steinberg, 1991; Parthasarathy et al., 1992). Oxidized low density lipoprotein cholesterol (LDL cholesterol) is taken up more readily by macrophages, which leads to the formation of foam cells and atherosclerotic plaques (Palinski et al., 1989). Mechanisms that slow or prevent this chain of events may decrease the risk of coronary heart disease (CHD) and stroke (Catapano, 1997). Flavonoids are a group of phenolic compounds and are known to have antioxidant properties (Kandaswani and Middleton, 1994). They have been reported to be scavengers of free radicals, including superoxide anions (Robak and Gryglewski, 1988), singlet oxygen (Husain et al., 1987), and lipid peroxy-radicals (Sorata et al., 1982). In addition, flavonoids have been shown to prevent LDL cholesterol oxidation and cytotoxicity in vitro (De Whalley et al., 1990).

According to Beretta et al. (2007), the experiments with endothelial cells fortified with the isolated fraction from native honey enriched in antioxidants, exposed to peroxyl radicals from 1,1-diphenyl-2-picrylhydrazyl (AAPH, $10 \mathrm{mM}$ ) and to hydrogen peroxide $\left(\mathrm{H}_{2} \mathrm{O}_{2}, 50-100\right.$ microM), indicated that phenolic acids and flavonoids were the main causes of the protective effect. They suggested that, through the synergistic action of its antioxidants, honey by reducing and removing ROS, may lower the risks and effects of acute and chronic free radical induced pathologies in vivo.

Rakha et al. (2008) showed that natural wild honey may exert its cardioprotective and therapeutic effects against epinephrine-induced cardiac disorders and vasomotor dysfunction directly, via its very pronounced total antioxidant capacity and its great wealth of both enzymatic and nonenzymatic antioxidants involved in cardiovascular defense mechanisms.

Nagyova et al. (2004) also demonstrated that short-term and modest supplementation with a mixture of antioxidant nutrients improves antioxidative capacity and reduces products of lipid peroxidation in plasma. Since a more pronounced effect 
was observed within the group of survivors of myocardial infarction, a recommendation of antioxidant supplements seems appropriate for patients with a history of cardiovascular disease.

Yochum et al. (1999) demonstrated that Flavonoids, a group of phenolic compounds, are known to have antioxidant properties. They prevent low density lipoprotein oxidation in vitro and thus may play a role in the prevention of coronary heart disease (CHD). In 1986, in a prospective study of 34,492 postmenopausal women in Iowa, the authors examined the association of flavonoid intake with CHD and stroke mortality. The data of this study suggested that flavonoid intake may reduce risk of death from CHD in postmenopausal women.

Another study by Xia et al. (2003) showed that the endothelium-derived vasoconstrictor endothelin-1 was increased after cardiopulmonary bypass in children with congenital heart defects. The study determined whether antioxidant therapy with Salvia miltiorrhiza injection, an herb extract containing phenolic compounds, prevents the postoperative increase of endothelin-1. They concluded that antioxidant therapy reduces myocardial damage and attenuates postoperative vasoactive mediator imbalance.

\section{Honey polyphenols for cardiovascular diseases}

Some of the polyphenols like quercetin, acacetin, caffeic acid phenethyl ester (CAPE), kaempferol, and galangin present in honey have been reported as promising pharmaceutical drugs in the treatment of cardiovascular diseases.

\section{Quercetin}

In a study (Yoshizumi et al., 2001) it has been proposed that daily intake of bioflavonoids belonging to polyphenols reduces the incidence of ischemic heart diseases (known as "French paradox"). It was hypothesized that bioflavonoids may affect Ang II-induced MAP kinase activation in cultured rat aortic smooth muscle cells (RASMC). The findings showed that Ang II stimulated rapid and significant activation of extracellular signal-regulated kinase (ERK) 1/2, c-Jun N-terminal kinase (JNK), and p38 in RASMC. Ang II-induced JNK activation was inhibited by 3,3',4',5,7-pentahydroxyflavone (quercetin), a major bioflavonoid in foods of plant origin, whereas ERK1/2 and p38 activation by Ang II were not affected by quercetin. Ang II caused a rapid tyrosine phosphorylation of Src homology and collagen (Shc), which was inhibited by quercetin. Quercetin also inhibited Ang II-induced Shc.p85 association and subsequent activation of phosphatidylinositol 3-kinase (PI3-K)/Akt pathway in RASMC. Furthermore, LY294002, a PI3-K inhibitor and a quercetin derivative, inhibited Ang II-induced JNK activation as well as Akt phosphorylation. Finally, Ang II-induced $[(3) \mathrm{H}]$ leucine incorporation was abolished by both quercetin and LY294002. These findings suggested that the preventive effect of quercetin on Ang II-induced VSMC hypertrophy are attributable, in part, to its inhibitory effect on Shc- and PI3-K-dependent JNK activation in VSMC. Thus, inhibition of JNK by quercetin may imply its usefulness for the treatment of cardiovascular diseases relevant to VSMC growth.

Several studies have found that chronic treatment with the dietary flavonoid quercetin lowers blood pressure and restores endothelial dysfunction in hypertensive animal models. Spontaneously hypertensive (male) rats (SHR) and Wistar-Kyoto (WKY) rats (5 weeks old) were treated with quercetin $(10 \mathrm{mg} / \mathrm{kg})$ or vehicle for 13 weeks. Changes in vascular expression of eNOS, caveolin-1 and p47 were analysed by Western blot, eNOS activity by conversion of $[\mathrm{H}]$ arginine to L-[H]citrulline, and NADPH oxidase activity by NADPH-enhanced chemoluminescence of lucigenin. In SHR, quercetin reduced the increase in blood pressure and heart rate and enhanced the endothelium-dependent aortic vasodilation induced by acetylcholine, but had no effect on the endothelium-independent response induced by nitroprusside. However, quercetin had no effect on endothelium-dependent vasoconstriction and aortic thromboxane B2 production. Compared to WKY, SHR showed upregulated eNOS and p47 protein expression, downregulated caveolin-1 expression, increased NADPH-induced superoxide production but, paradoxically, eNOS activity was reduced. Chronic quercetin treatment prevented all these changes in SHR. In WKY, quercetin had no effect on blood pressure, endothelial function or the expression or activity of the proteins analysed. Enhanced eNOS activity and decreased NADPH oxidase-mediated superoxide anion $\left(\mathrm{O}_{2}\right)$ generation associated with reduced p47 expression appear to be essential mechanisms for the improvement of endothelial function and the antihypertensive effects of chronic quercetin (Sánchez et al., 2006).

In another study, Carlstrom et al. (2007) showed that diets high in quercetin may decrease the risk of developing cardiovascular disease. They tested whether quercetin delays or reduces the severity of hypertension, vascular dysfunction, or cardiac hypertrophy in the spontaneously hypertensive rat (SHR). Normotensive, 5-wk-old SHR consumed standard (n 1/4 18) or quercetin-supplemented diet (1.5 g quercetin/kg diet, $\mathrm{n} 1 / 422$, SHR-Q) for 5 or 11 wks. Wistar Kyoto rats (WKY, n 1/4 19), fed a standard diet, served as controls. At $16 \mathrm{wk}$, plasma quercetin, measured by HPLC, was $2.0960 .33 \mathrm{mmol} / \mathrm{L}$ in SHR-Q and below assay detection limits in SHR and WKY rats. At 10 and 16 wk of age, arterial blood pressure and heart weight:body weight were not different between SHR and SHR-Q. At $16 \mathrm{wk}$, cardiac function (echocardiography), vascular morphology (hematoxylin and eosin staining of aortae), and resistance and conductance vessel reactivity (wire myography) were unchanged in SHR vs. SHR-Q. Thus, a quercetin supplemented diet does not delay the onset or lessen the severity of cardiovascular complications that develop in SHR. The efficacy of quercetin depends on its method of delivery, 15 -wk-old SHR were given quercetin $(10 \mathrm{mg} / \mathrm{kg}) \mathrm{once}$ daily via oral gavages for 4 consecutive days. Arterial blood pressure ( $\mathrm{mm} \mathrm{Hg}$ ) was lower in gavaged SHR (148 6 5) than in SHR-Q (162 $62, \mathrm{P}, 0.02)$ and SHR (168 $63, \mathrm{P}, 0.001)$ These data suggested that mode of delivery is a critical determinant in whether quercetin provides cardiovascular benefits.

In another study, the effects of an oral daily dose $(10 \mathrm{mg} \mathrm{kg} 71)$ of the avonoid quercetin for 5 weeks in spontaneously hypertensive (SHR) and normotensive Wistar Kyoto rats (WKY) were analysed. Quercetin induced a significant reduction in 
systolic $(718 \%)$, diastolic $(723 \%)$ and mean $(721 \%)$ arterial blood pressure and heart rate $(712 \%)$ in SHR but not in WKY rats. The left ventricular weight index and the kidney weight index in vehicle-treated SHR were significantly greater than in control WKY and these parameters were significantly reduced in quercetin-treated SHR in parallel with the reduction in systolic blood pressure. Quercetin had no effect on the vasodilator responses to sodium nitroprusside or to the vasoconstrictor responses to noradrenaline or $\mathrm{KCl}$ but enhanced the endothelium-dependent relaxation to acetylcholine $(\mathrm{Emax}=58+5 \% \mathrm{vs} 78+5 \%$, in isolated aortae. The $24 \mathrm{~h}$ urinary isoprostane F2a excretion and the plasma malonyldialdehyde (MDA) levels in SHR rats were increased as compared to WKY rats. In quercetin-treated SHR rats, both parameters were similar to those of vehicle-treated WKY. These data demonstrated that quercetin reduces the elevated blood pressure, the cardiac and renal hypertrophy and the functional vascular changes in SHR rats without effect on WKY. These effects were associated with a reduced oxidant status due to the antioxidant properties of the drug (Duarte et al., 2001).

Epidemiological studies reported that quercetin, an antioxidant flavonol is associated with reduced risk of coronary heart disease and stroke. Quercetin supplementation also reduces blood pressure in hypertensive rodents. The efficacy of quercetin supplementation to lower blood pressure in hypertensive humans has never been evaluated. This study tested the hypothesis that quercetin supplementation reduces blood pressure in hypertensive patients and then it has been determined whether the antihypertensive effect of quercetin is associated with reductions in systemic oxidant stress. Men and women with prehypertension (n 1/4 19) and stage 1 hypertension (n 1/4 22) were enrolled in a randomized, double-blind, placebo-controlled, crossover study to test the efficacy of $730 \mathrm{mg}$ quercetin/d for $28 \mathrm{~d}$ vs. placebo. Blood pressure (mm Hg, systolic/diastolic) at enrollment was $13762 / 8661$ in prehypertensives and $14862 / 9661$ in stage 1 hypertensive subjects. Blood pressure was not altered in prehypertensive patients after quercetin supplementation. In contrast, reductions in $(\mathrm{P}, 0.01) \mathrm{systolic}(2762 \mathrm{mmHg})$, diastolic (25 $62 \mathrm{mmHg}$ ), and mean arterial pressures $(2562 \mathrm{~mm} \mathrm{Hg}$ ) were observed in stage 1 hypertensive patients after quercetin treatment. However, indices of oxidant stress measured in the plasma and urine were not affected by quercetin. These data showed that quercetin supplementation reduces blood pressure in hypertensive subjects. Contrary to animal-based studies, there was no quercetin-evoked reduction in systemic markers of oxidative stress (Randi et al., 2007)

\section{Acacetin}

Gui-Rong et al. (2008) investigated whether the natural flavone acacetin would be an atrium-selective anti-atrial fibrillation agent. The effects of acacetin on human atrial ultrarapid delayed rectifier $\mathrm{K}^{+}$current $\left(I_{\text {Kur }}\right)$ and other cardiac ionic currents were studied with a whole-cell patch technique. Acacetin suppressed $I_{\mathrm{Kur}}$ and the transient outward $\mathrm{K}^{+}$current (IC 503.2 and $9.2 \mu \mathrm{mol} / \mathrm{L}$, respectively) and prolonged action potential duration in human atrial myocytes. The compound blocked the acetylcholine-activated $\mathrm{K}^{+}$current; it had no effect on the $\mathrm{Na}^{+}$current, L-type $\mathrm{Ca}^{2+}$ current, or inward-rectifier $\mathrm{K}^{+}$current in guinea pig cardiac myocytes. Although acacetin caused a weak reduction in the hERG and hKCNQ1/hKCNE1 channels stably expressed in HEK 293 cells, it did not prolong the corrected QT interval in rabbit hearts. In anesthetized dogs, acacetin (5 mg/kg) prolonged the atrial effective refractory period in both the right and left atria 1 to $4 \mathrm{hrs}$ after intraduodenal administration without prolongation of the corrected QT interval, whereas sotalol at $5 \mathrm{mg} / \mathrm{kg}$ prolonged both the atrial effective refractory period and the corrected QT interval. Acacetin prevented atrial fibrillation (AF) induction at doses of $2.5 \mathrm{mg} / \mathrm{kg}(50 \%), 5 \mathrm{mg} / \mathrm{kg}(85.7 \%)$, and 10 $\mathrm{mg} / \mathrm{kg}(85.7 \%)$. Sotalol $5 \mathrm{mg} / \mathrm{kg}$ also prevented AF induction (60\%). The study demonstrated that the natural compound acacetin is an atrium-selective agent that prolongs the atrial effective refractory period without prolonging the corrected QT interval and effectively prevents AF in anesthetized dogs after intraduodenal administration. These results have indicated that oral acacetin is a promising atrium-selective agent for the treatment of AF.

\section{Caffeic acid}

Caffeic acid phenethyl ester (CAPE) is a phenolic active component of propolis of honeybee hives and reduces heart rate and blood pressure in rats. Iraz et al. (2005) have investigated the role of vagal activity and atropine blockage on the bradycardic and hypotensive effects of CAPE in rats. The rats were divided into five groups $(\mathrm{n}=8)$. Saline and vehicle $(10 \%$ ethanol) of CAPE were given to the first and second groups, respectively. Group 3 was treated with $5 \mathrm{mg} / \mathrm{kg}$ CAPE. Group 4 bivagotomized and treated with $5 \mathrm{mg} / \mathrm{kg}$ CAPE. Group 5 treated with atropine $(5 \mathrm{microg} / \mathrm{microL} / \mathrm{min})$ continuously and treated with CAPE. The electrophysiological monitoring was done for each experiment under urethane anesthetia. As a result, CAPE caused intense and transient bradycardia and hypotension. Vagotomy completely abolished bradycardia which occurred via CAPE injection; however atropine attenuated bradycardic effects of CAPE. On the other hand, hypotensive effect of CAPE was affected from neither bilateral vagotomy nor atropine treatment. It was thought that CAPE may exert its effects on heart rate via a central parasympathetic control mechanism, but not on central parasympathetic blood pressure control system.

\section{Kaempferol}

$\mathrm{Xu}$ et al. (2006) investigated the vascular effects of kaempferol in isolated porcine coronary artery rings. U46619 (9,11dideoxy-9 $\alpha, 11 \alpha$-methanoepoxy prostaglandin $\mathrm{F} 2 \alpha, 30 \mathrm{nM}$ ) was used to contract porcine coronary arterial rings. Concentration relaxation curve of kaempferol $(1 \mathrm{nM}-100 \mu \mathrm{M})$ was constructed and kaempferol demonstrated significant relaxation at high concentrations. However, at low concentration, kaemferol has no significant effect on relaxation. Kaempferol $(10 \mu \mathrm{M})$ enhanced relaxation produced by bradykinin, the calcium ionophore A23187, isoproterenol and sodium nitroprusside in endothelium-intact porcine coronaryarteries. 
In endothelium-disrupt rings, kaempferol $(10 \mu \mathrm{M})$ also enhanced the relaxation caused by isoproterenol, sodium nitroprusside, levcromakalim and nifedipine. On the other hand, antioxidant agents did not affect bradykinin-induced relaxation or the enhancement effect of kaempferol. It was concluded that a low concentration of kaempferol $(10 \mu \mathrm{M})$, devoid of significant vascular effect, has the ability to enhance endothelium-dependent and endothelium-independent relaxations. This action of kaempferol is unrelated to its antioxidant property.

Another study examined whether or not the ER stress and Bcl-2 proteins are linked to the protective effect of kaempferol, a phytoestrogen, on ischemia-reperfusion (I/R)-induced cardiac damage. In order to determine if kaempferol modifies the I/R-induced response in $\mathrm{H} 9 \mathrm{c} 2$ cardiac muscle cells, the cells were exposed to kaempferol followed by ischemia $12 \mathrm{~h} /$ reperfusion $4 \mathrm{~h}$. kaempferol had a protective effect on the apoptosis induced by $\mathrm{I} / \mathrm{R}$ in the cardiac muscle cells. The kaempferol treatment significantly increased the expression level of the anti-apoptotic protein, Bcl-2, but decreased the level of the pro-apoptotic protein, bax. Kaempferol down-regulated the expressions of the endoplasmic reticulum (ER) stress proteins, GRP78, ATF-6alpha, XBP-2, IRE1-alpha, phosphor-eIF-2alpha and CHOP. In ex vivo-Langendorff experiment, the kaempferol treatment regulated the expression of ER stress proteins-CHOP and GRP78. The kaempferol also improved the post-ischemic LVEDP and LVDP significantly after 20,30, 40 and 50 min of reperfusion compared with the untreated control hearts, which showed that kaempferol offers protection against I/R-associated cardiac dysfunction (Kim et al., 2008).

\section{Galangin}

Galangin has anti-oxidative effect on endothelial tissues, thus affects lipid peroxidation and can prevent heart disease)Therefore, it helps to preserve other protective antioxidants such as vitamin E, vitamin C, and other flavonoids, and also can prevent lipid peroxidation (Lysias-Derrida, 2006).

\section{Conclusion}

Antioxidants present in honey come from a variety of sources, and include Vitamin C, monophenolics, flavonoids, and polyphenolics. Regular flavonoid intake is associated with a reduced risk of cardiovascular diseases. In the coronary heart disease, the protective effects of flavonoids include mainly antithrombotic, antiischemic, antioxidant, and vasorelaxant and flavonoids decrease the risk of coronary heart disease by three major actions: (a) improving coronary vasodilatation, (b) decreasing the ability of platelets in the blood to clot, and (c) preventing LDLs from oxidizing. Although there is a wide spectrum of polyphenols types, quercetin, caffeic acid phenethyl ester, acacetin, kaempferol, galangin, predominate in many honeys. This review has clearly demonstrated that certain honey polyphenols have a promising pharmacological role in preventing cardiovascular diseases. After generating more in-depth and exhaustive information of these compounds jointly in vitro and in vivo studies, clinical trials should be initiated to further validate these compounds in medical applications.

\section{References}

1. Aljadi, A.M. and Kamaruddin, M.Y. (2004). Evaluation of the phenolic contents and antioxidant capacities of two Malaysian floral honeys. Food Chem., 85: 513-518.

2. Al-Waili, N.S. (2003). Effects of daily consumption of honey solution on hematological indices and blood levels of minerals and enzymes in normal individuals. J. Med. Food., 6: 135-140.

3. Andrade, P., Ferreres, F. and Amaral, M.T. (1997). Analysis of honey phenolic acids by HPLC, its application to honey botanical characterization. J. Liq. Chromatogr. Relat. Technol., 20: 2281-2288.

4. Baltrusaityte, V., Venskutonis, P.R. and Ceksteryte, V. (2007). Radical scavenging activity of different floral origin honey and beebread phenolic extracts. Food Chem., 101: 502-514.

5. Benavente-Garcı'a, O., Castillo, J. and Marı'n, F.R. (1997). Uses and properties of Citrus flavonoids. J. Agric. Food. Chem., 45: 4505-4515.

6. Beretta, G., Granata, P. and Ferrero, M. (2005). Standardization of antioxidant properties of honey by a combination of spectrophotometric/fluorimetric assays and chemometrics. Anal. Chim. Acta., 533: 185-191.

7. Beretta, G., Orioli, M. and Facino, R.M. (2007). Antioxidant and radical scavenging activity of honey in endothelial cell cultures (EA.hy926). Planta Med., 73(11): 1182-1189. Epub 2007 Sep 7.

8. Blasa, M., Candiracci, M. and Accorsi, A. (2006). Raw Millefiori honey is packed full of antioxidants. Food. Chem. 97: 217-222.

9. Bravo, L. (1998). Polyphenols: chemistry, dietary sources, metabolism, and nutritional significance. Nutr. Rev., 56: 317-333.

10. Carlstrom, J., Symons, D., Ching, T., Wu, Bruno, R. S., Sheldon, E., Litwin, and Jalili, T. (2007). A Quercetin Supplemented Diet Does Not Prevent Cardiovascular Complications in Spontaneously Hypertensive Rats1. J. Nutr., 137: 628-633.

11. Catapano, A.L. (1997). Antioxidant effect of flavonoids. Angiology, 48: 39-44.

12. Cherchi, A., Spanedda, L., Tuberoso, C. and Cabras, P. (1994). Solid-phase extraction of high-performance liquid chromatographic determination of organic acids in honey. J. Chromatogr., 669: 59-64.

13. D'Arcy, B.R. (2005). Antioxidants in Australian floral honeys -Identification of health enhancing nutrient components. RIRDC Publication No 05/040, 1

14. De Whalley, C.V., Rankin, S.M. and Hoult, J.R.S. (1990). Flavonoids inhibit the oxidative modification of low-density lipoproteins by macrophages. Biochem. Pharmacol., 39: 1743-1750.

15. Depeint, F., Gee, J.M., Williamson, G. and Johnson, I.T. (2002). Evidence for consistent patterns between flavonoid structures and cellular activities. Proc. Nutr. Soc., 61: 97-103. 
16. Duarte, J., PeÂ rez-Palencia, R., Vargas, F., Ocete, M. A., Pê̂A rez-Vizcaino, F., Zarzuelo, A. and Tamargo, J. (2001). Antihypertensive effect of the avonoid quercetin in spontaneously hypertensive rats. Br. J. Pharmacol., 133: $117-124$.

17. Fahey, J.W. and Stephenson, K.K. (2002). Pinostrobin from honey and Thai ginger (Boesenbergia pandurata): A potent flavonoid inducer of mammalian phase 2 chemoprotective and antioxidant enzymes. J. Agric. Food. Chem., 50: 7472-7476.

18. Ferreres, F., Ortiz, A. and Silva, C. (1992). Flavonoids of "La Alcarria" honey. Z Lebensm Unters Forsch. 194: 139-143.

19. Frankel, S., Robinson, G.E. and Berenbaum, M.R. (1998). Antioxidant capacity and correlated characteristics of 14 unifloral honeys. J. Apic. Res., 37: 27-31.

20. Garc'ia, B. and Castillo, J. (2008). Update on Uses and Properties of Citrus Flavonoids: New Findings in Anticancer, Cardiovascular, and Anti-inflammatory Activity J. Agric. Food Chem. 56: 6185-6205

21. Gheldof, N. and Engeseth, N.J. (2002). Antioxidant capacity of honeys from various floral sources based on the determination of oxygen radical absorbance capacity and inhibition of in vitro lipoprotein oxidation in human serum samples. J. Agric. Food Chem., 50: 3050-3055.

22. Gheldof, N., Wang, X.H. and Engeseth, N.J. (2003). Buckwheat honey increases serum antioxidant capacity in humans. J. Agric. Food Chem., 51: 1500-1505.

23. Gui-Rong L., Wang, H.B., Qin, G.W., Jin, M.W., Tang, Q., Sun, H.Y., Du, X.L., Deng, X.L., Zhang, X.H., Chen, J.B. and Chen L. (2008). Acacetin, a Natural Flavone, Selectively Inhibits Human Atrial Repolarization Potassium Currents and Prevents Atrial Fibrillation in Dogs. Circulation., 117: 2449-2457.

24. Guzik, T.J., West, N.E., Black, E., McDonald, D., Ratnatunga, C., Pillai, R. and Channon, K.M. (2000). Vascular superoxide production by $\mathrm{NAD}(\mathrm{P}) \mathrm{H}$ oxidase: Association with endothelial dysfunction and clinical risk factors. Circ. Res., 86: E85-E90.

25. Hertog, M.G., Feskens, E.J. and Hollman, P.C. (1993). Dietary antioxidant flavonoids and risk of coronary heart disease: the Zutphen Elderly Study. Lancet., 342: 1007-1011.

26. Hink, U., Li, H., Mollnau, H., Oelze, M., Matheis, E., Hartmann, M., Skatchkov, M., Thaiss, F., Stahl, R.A., Warnholtz, A., Meinertz, T., Griendling, K., Harrison, D.G., Forstermann, U. and Munzel, T. (2001). Mechanisms underlying endothelial dysfunction in diabetes mellitus. Circ. Res., 88: E14-E22.

27. Husain, S.R., Cillard, J. and Cillard, P. (1987). Hydroxy radical scavenging activity of flavonoids. Phytochemistry, 26: 24892492.

28. Inoue, K., Murayarna, S. and Seshimo, F. (2005). Identification of phenolic compound in manuka honey as specific superoxide anion radical scavenger using electron spin resonance (ESR) and liquid chromatography with coulometric array detection. J. Sci. Food. Agric., 85: 872-878.

29. Iraz, M., Fadillioglu, E., Tasdemir, S. and Erdogan, S. (2005). Role of vagal activity on bradicardic and hypotensive effects of caffeic acid phenethyl ester (CAPE). Cardiovasc. Toxicol., 5(4): 391-396.

30. Jaganathan, S. K. and Mandal, M. (2009). Antiproliferative Effects of Honey and of Its Polyphenols: A Review. Hindawi Publishing Corporation, Journal of Biomedicine and Biotechnology, Article ID 830616, 13 pages doi:10.1155/2009/830616.

31. Jendekova, L., Kojsova, S., Andriantsitohaina, R. and Pechanova, O. (2006). The time-dependent effects of Provinols on brain NO synthase activity in L-NAME-induced hypertension. Physiol. Res. 55: S31-S37.

32. Kandaswani, C. and Middleton, E. (1994). Free radical scavenging and antioxidant activity of plant flavonoids. Adv. Exp. Med. Biol.,336: 351-376.

33. Kim, D.S., Ha, K.C., Kwon, D.Y., Kim, M.S., Kim, H.R., Chae, S.W. and Chae, H.J.(2008). Kaempferol Protects Ischemia/Reperfusion-Induced Cardiac Damage Through the Regulation of Endoplasmic Reticulum Stress. Immunopharmacology and Immunotoxicology. 30(2): 257-270.

34. Kücük, M., Kolayli, S., Karaoglu, S., Ulusoy, E., Baltaci, C. and Candan, F. (2007). Biological activities and chemical composition of three honeys of different types from Anatolia. Food Chem., 100: 526-534.

35. Lysias-Derrida, C. (2006). Galangal: Lesser galangal [on-line]. Available: http://www.mdidea.com/products/new/-new003.html

36. Marchend, L.L. (2002). Cancer preventive effects of flavonoids: A review. Biomed. Pharmacother., 56: $296-301$.

37. Martınez, C., Yanez, J. and Alcaraz, M. (2003). Effects of several polyhydroxylated flavonoids on the growth of B16F10 melanoma and Melan-a cell lines. Influence of sequential oxidation state on the flavonoid skeleton. Melanoma. Res., 13: 3-9.

38. Middleton, E., Kandaswami, C. and Theoharides, T.C. (2000). The effects of plant flavonoids on mammalian cells: Implications for inflammation, heart disease and cancer. Pharmacol. Rev. 52: 673-751.

39. Miller, F. J., Jr., Gutterman, D. D., Rios, C. D., Heistad, D. D. and Davidson, B. L. (1998). Superoxide production in vascular smooth muscle contributes to oxidative stress and impaired relaxation in atherosclerosis. Circ. Res., 82: 1298-1305.

40. Morawietz, H., Weber, M., Rueckschloss, U., Lauer, N., Hacker, A. and Kojda, G. (2001). Upregulation of vascular NAD(P)H oxidase subunit gp91phox and impairment of the nitric oxide signal transduction pathway in hypertension. Biochem. Biophys. Res.Commun., 85: 1130-1135.

41. Mugge, A., Brandes, R.P., Boger, R.H., Dwenger, A., Bode-Boger, S., Kienke, S., Frolich, J.C. and Lichtlen, P.R. (1994). Vascular release of superoxide radicals is enhanced in hypercholesterolemic rabbits. J. CardioVasc. Pharmacol., 24: 994-998.

42. Nagai, T., Inoue, R., Kanamori, N., Suzuki, N. and Nagashima, T. (2006). Characterization of honey from different floral sources. Its functional properties and effects of honey species on storage of meat. Food. Chem. 97: 256-262.

43. Nagyova. A., Krajcovicova-Kudlackova, M., Horska, A., Smolkova, B., Blazicek, P., Raslova, K., Collins, A. and Dusinska, M. (2004). Lipid peroxidation in men after dietary supplementation with a mixture of antioxidant nutrients. Bratisl Lek Listy, 105(78): $277-80$.

44. National Honey Board "Food Technology Program" [cited 2009 Sep 12] Available from: http://www.aaccnet.org/funcfood/content/releases/Honey-antioxidant.htm

45. National Honey Board, Honey and Bees, (2007). [cited 2009 Sep 10] Available from: http://www.honey.com/consumers/kids/beefacts.asp.

46. Palinski, W., Rosenfeld, M.E. and Yla-Herttuala, S. (1989). Low density lipoprotein undergoes oxidative modification in vivo. Proc. Natl. Acad. Sci. U S A, 86: 1372-1376. 
47. Parthasarathy, S., Steinberg, D. and Witztum, J.L. (1992). The role of oxidized low-density lipoproteins in the pathogenesis of atherosclerosis. Ann. Rev. Med., 43: 219-225.

48. Perez, R.A., Iglesias, M.T., Pueyo, E., Gonzalez, M., de Lorenzo, C. (2007). Amino acid composition and antioxidant capacity of Spanish honeys. J. Agric. Food. Chem., 55: 360-365.

49. Rakha, M.K., Nabil, Z.I. and Hussein, A.A. (2008). Cardioactive and vasoactive effects of natural wild honey against cardiac malperformance induced by hyperadrenergic activity. J. Med. Food., 11(1): 91-98.

50. Randi, L., Edwards, Lyon, T., Litwin, S. E., Rabovsky, A., Symons, J. D. and Jalili, T. (2007). Quercetin Reduces Blood Pressure in Hypertensive Subjects. J. Nutr., 137: 2405-2411.

51. Renaud, S. and Lorgeril, M.D. (1992). Wine, alcohol, platelets and the French paradox for coronary heart disease. Lancet; 339: 1523-1526.

52. Robak, J. and Gryglewski, R.J. (1988). Flavonoids are scavengers of superoxide anion. Biochem. Pharmacol., 37: 83-88.

53. Rodriguez, J., Yanez, J. and Vicente, V. (2002). Effects of several flavonoids on the growth of B16F10 and SK-MEL-1 melanoma cell lines: Relationship between structure and activity. Melanoma. Res., 12: 99-107.

54. Sánchez, M., Galisteo, M., Vera, R., Villar, I.C., Zarzuelo, A., Tamargo, J., Pérez-Vizcaíno, F. and Duarte, J. (2006). Quercetin downregulates NADPH oxidase, increases eNOS activity and prevents endothelial dysfunction in spontaneously hypertensive rats. J. Hypertens., 24(1): 75-84.

55. Sano, T., Umeda, F., Hashimoto, T., Nawata, H. and Utsumi, H. (1998). Oxidative stress measurement by in ViVo electron spin resonance spectroscopy in rats with streptozotocin-induced diabetes. Diabetologia, 41: 1355-1360.

56. Schramm, D.D., Karim, M., Schrader, H.R., Holt, R.R., Cardetti, M., Keen and C.L. (2003). Honey with high levels of antioxidants can provide protection to healthy human subjects. J. Agric. Food Chem., 51: 1732-1735.

57. Serafini, M., Ghiselli, A. and Ferro-Luzzi, A. (1994). Red wine, tea and antioxidants. Lancet, 344: 626-630.

58. Sorata, Y., Takahama, U. and Kimura, M. (1982). Protective effect of quercetin and rutin on photosensitized lysis of human erythrocytes in the presence of hematoporphyrin. Biochem. Biophys. Acta.,799: 313-317.

59. Suzuki, H., Swei, A., Zweifach, B. W. and Schmid-Schonbein, G. W. (1995). In vivo evidence for microvascular oxidative stress in spontaneously hypertensive rats. Hydroethidine microfluorography. Hypertension, 25: 1083-1089.

60. Tan, S.T., Wilkins, A.L., Holland, P.T. and McGhie, T.K. (1989). Extractives from New Zealand unifloral honeys. 2. Degraded carotenoids and other substances from heather honey. J. Agric. Food. Chem., 37: 1217-1221.

61. Vela, L., de Lorenzo, C. and Pérez, R.A. (2007). Antioxidant capacity of Spanish honeys and its correlation with polyphenol content and other physicochemical properties. J. Sci. Food Agric., 87: 1069-1075.

62. White, J.W. (1975). Composition of honey. En: Crane E, ed. Honey, a comprehensive survey. London: Bee research Association and Chalfont St Peter, 157-206.

63. White, J.W. and Rudyj, O.N. (1978). The protein content of honey. J. Apic. Res., 17: 234-238.

64. Witztum, J.L., Steinberg, D. (1991). Role of oxidized low density lipoprotein in atherogenesis. J. Clin. Invest., 88: $1785-1792$.

65. Xia, Z., Gu, J., Ansley, D. M., Xia, F. and Yu, J. (2003). Antioxidant therapy with Salvia miltiorrhiza decreases plasma endothelin-1 and thromboxane B2 after cardiopulmonary bypass in patients with congenital heart disease. J. Thorac. Cardiovasc. Surg., 126(5): 1404-1410.

66. Xu, Y.C., Yeung, D.K.Y., Man, R.Y.K. and Leung, S.W.S. (2006). Kaempferol enhances endothelium-independent and dependent relaxation in the porcine coronary artery. Mol. Cell. Biochem., 287: 61-67.

67. Yanez, J., Vicente, V. and Alcaraz, M. (2004). Cytotoxicity and antiproliferative activities of several phenolic compounds against three melanocytes cell lines: Relationship between structure and activity. Nutr. Cancer., 49: 191-199.

68. Yochum, L., Lawrence, H., Kushi, Katie Meyer, and Folsom, A. R. (1999). Dietary Flavonoid Intake and Risk of Cardiovascular Disease in Postmenopausal Women. Am. J. Epidemiol. 149: 943-949.

69. Yoshizumi, M., Tsuchiya, K., Kirima, K., Kyaw, M., Suzaki, Y. and Tamaki, T. (2001). Quercetin inhibits Shc- and phosphatidylinositol 3-kinase-mediated c-Jun N-terminal kinase activation by angiotensin II in cultured rat aortic smooth muscle cells. Mol. Pharmacol., 60(4): 656-665.

70. Zalba, G., Beaumont, F.J., San Jose, G., Fortuno, A., Fortuno, M.A., Etayo, J.C. and Diez, J. (2000). Vascular NADH/NADPH oxidase is involved in enhanced superoxide production in spontaneously hypertensive rats. Hypertension, 35: 1055-1061. 